\title{
A Ubiquitous Solution for Location-Aware Games
}

\author{
André Pinto ${ }^{1,3}$, António Coelho ${ }^{1,2,3}$, and Hugo da Silva ${ }^{1,2}$ \\ 1 Departamento de Engenharia Informática, Faculdade de Engenharia, \\ Universidade do Porto, Porto, Portugal \\ \{andre.da.silva.pinto, acoelho, hugo.da.silva\}@fe.up.pt \\ 2 INESC TEC (formerly INESC Porto), Porto, Portugal \\ 3 3Decide, Palcos da Realidade, Porto, Portugal
}

\begin{abstract}
Even though we now witness a popular use of location-based mobile games, the player experience in these applications is always limited by the errors of common location technologies, especially in indoor scenarios. This paper describes the way we minimize this problem in our game development platform, by levering the potential behind smartphone sensors to estimate players' trajectories. Our approach is based on a Pedestrian Dead Reckoning (PDR) algorithm that combines methods to determine orientation, detect steps and estimate their length. Other typical multiplayer mobile games problems, like network latency, are also briefly addressed.
\end{abstract}

Keywords: location-based games, mobile games, location-based services, ubiquitous, indoor, pedestrian dead reckoning.

\section{Introduction}

During the last years, the proliferation of smartphones with location capabilities enabled the creation of several categories of location-based services (LBS). Among them, the mobile social gaming phenomena ended up attracting more attention to the opportunities created by these technological advances in the entertainment field. At the same time, ubiquitous computing, a model in which information is omnipresent and contextualized, is now more prevalent than ever before [1].

Although enabling interesting use cases for mobile games, everyone who used a GPS-enabled device, has certainly experienced its accuracy and availability bottlenecks, whether caused by indoor usage, city canyons or other physical obstacles. Even the alternative solutions, like WLAN positioning, can get its performance critically deteriorated in dynamic non-line-of-sight situations. These limitations are restraining mobile games (and many other LBS) from exploring truly ubiquitous experiences.

The solution presented in this paper was motivated by the development of a serious game that helps the player to explore a faculty building by engaging the user in a "treasure hunt". During the gameplay the player gets to know the most 


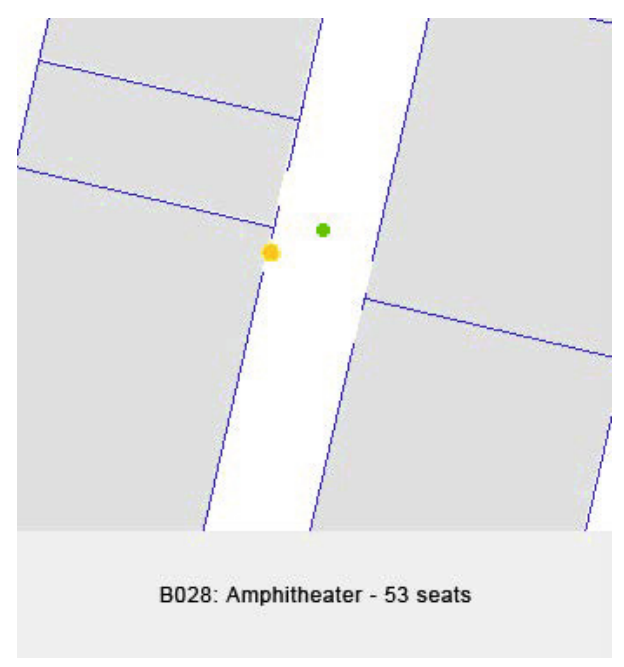

Fig. 1. Game screen showing the player (green) near a point of interest (yellow)

important places around him/her, whilst receiving basic useful information of the surroundings (Figure 1). By visiting specific points of interest within the faculty campus, the player collects points for his/her team. This use case implies some technical requirements such as indoor player positioning and intensive location reporting to the server.

To enable the development of this type of games, we propose a multiplayer mobile gaming platform that was developed to diminish the above inaccuracies by implementing a pedestrian dead reckoning method that uses the sensors usually available on recent smartphones (accelerometers, magnetometers and gyroscopes) to estimate the players' positions. Besides the location problem, our platform also offers a basic communication system created to minimize latency impact during the necessary data exchange in a multiplayer environment.

\section{Platform Architecture}

Our platform is composed by several client applications (one per player), which access one server application by using the available network technology in the place (e.g. WLAN, 3G, 4G). Both of these applications are written in Java. The client applications are responsible for reporting the player position to the server and engaging the user in the game mechanics. The server application manages the game communication and controls game-sensitive data, like the scoreboard. Finally there is a third entity, a PostGIS database, from which the client applications obtain the building plan data. In our test case, this data is used to render the plan and show information about the points of interest near the player. 
Although we had used a Sparkfun Razor IMU with 9 Degrees of Freedom to gather the sensors data, the approach is equality valid for any smartphone which fulfil the necessary hardware requirements.

\section{Related Work}

The pedestrian dead reckoning approaches to location is not a new topic in the scientific community. From the various work already done, we highlight the 1996's patent [2, which provides an initial overview of the solution as a whole, and, more recently [3], that offers an implementation for handheld devices. Other solutions are also addressed in surveys like 4 and [5], which enable the reader to better understand the complete panorama of indoor location methods. On the other hand, communication problems like network latency, which are common in these situations, have also been targeted in $[6$.

\section{Pedestrian Dead Reckoning (PDR)}

PDR is a method used by Inertial Navigation Systems. In this kind of systems, the positioning process is based on a relative estimation. The previous known position of the subject/object's is used together with an estimate for its displacement to obtain an approximation of the current position. For PDR solutions, the displacement estimate intends to measure the distance of pedestrian locomotion. The method is composed by 3 main tasks:

- orientation determination,

- step detection,

- step length estimation.

In a "disturbance-free environment", orientation could be given directly by the magnetometer's readings. In the "real world", magnetic fields are very irregular which makes the former method a naive use of this sensor. On the other hand, electronic gyroscopes have offset and drift errors, so they can't be a reliable source for orientation without realizing further steps to treat their data. In order to fix this we combined the gyroscope's data with the values received from the magnetometers and accelerometers into a direction cosine matrix. This process includes several different methods that are generically described in [7]. Still, this process is not free from the negative effects of magnetic disturbances, so we implemented an additional technique to detect interferences on the magnetometer's signal [8]. We did this by monitoring sudden changes in the norm of the magnetic field, and temporarily ignoring the magnetometer's data while they are present.

The step detection process is based on a state machine that processes the accelerometer signal over the vertical axis and detects "step-like" waves. The state machine contains 4 states: IDLE, ACC UP, ACC DOWN and STOPPING. Each one of these states has its own transition conditions that reflect the mechanic of the different step phases. 


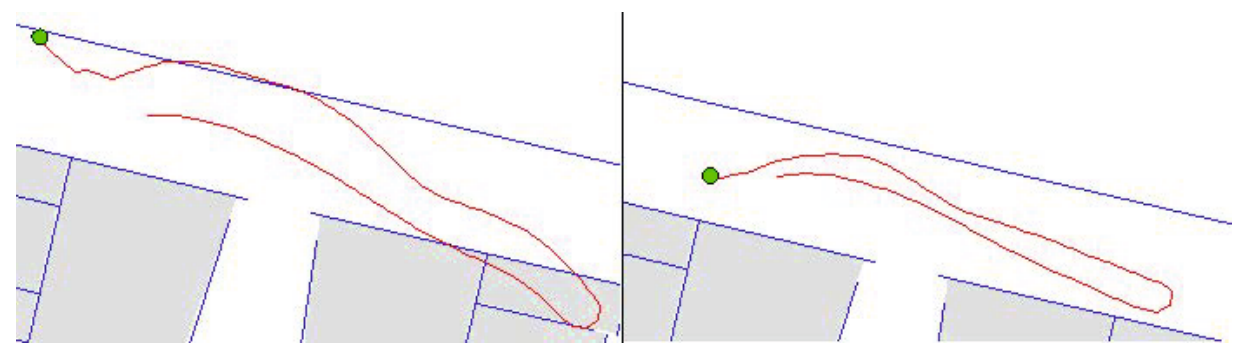

Fig. 2. Player's straight line trajectory (red) obtained by using our approach without (left) and with (right) magnetic disturbances detection

Finally, as a step length depends on multiple factors that can be associated with the subject (e.g. height, sex), the environment (e.g. inclinations, stairs) or the walk dynamics (e.g. step frequency and walk gait), we used various different metrics obtained from the accelerometer to estimate its value, namely: the double integral of acceleration's norm (without gravity), the maximum amplitude of vertical acceleration, the minimum anteroposterior acceleration and a step frequency estimate. An additional constant factor is added to represent a reference value. During an initial calibration phase, the weights $\left(w_{i}\right)$ of each one of these factors $\left(v_{i}\right)$ are estimated by using multiple linear regression. Afterwards they are combined to calculate the step length $(s L)$ estimate for every detected step:

$$
s L=\sum_{i=1}^{5} w_{i} \cdot v_{i}
$$

\section{Communication}

There is a strong relation between the communication requirements and the game genre. When creating a platform for mobile serious games, it is not possible to predict the kind of games it will be used for, so the optimization work was focused on the system's reaction to the worst-case scenario. Many of the problems present in the worst-case scenario are greatly increased by mobile network characteristics, which are typically slower and more error-prone than the wired networks. This easily leads to package loss and latency. Considering the circumstances, different protocols (UDP and TCP) were assessed and ways to minimize the negative effects of latency (e.g. dead reckoning) were studied.

For location-based games that heavily rely on a continuous feed of messages reporting the player's position, the importance of a single message is very low as it becomes immediately outdated with the next one in the stream. Therefore, a protocol like TCP, whose methods for granting reliability introduce considerable delay on the network, is unsuited to our case. For instance, if one position update message fails to reach its target, skipping it and sending the new position instead, would be a better option than trying to send the obsolete position again. 
Additionally, the bigger segment header (UDP: 8 bytes, TCP: 20 bytes) and the considerable weight of acknowledgement messages in the network transfers $(38 \%$ 9]), are all strong drawbacks for TCP in its comparison with UDP. Thus we ended up using UDP to handle the communication between the different actors. Our conclusions are also supported by [6] where UDP invariably outperforms TCP in every tested network (e.g. UDP UMTS: $458 \mathrm{~ms}$ avg, TCP UMTS: 933 ms avg).

\section{Conclusions and Future Work}

Overall, the final solution produced encouraging results when compared with the naive approach, but even with all the previous corrections, the PDR displacement estimation's accuracy still depends on the sensors data reliability. As can be seen in Figure 2, our approach creates some deviation from the real trajectory. This is especially worrying, as these errors accumulate in each iteration (as is typical in INS), which can lead to useless estimates relatively quickly. In order to minimize this accumulated error we plan to implement restrictions on the possible trajectories performed by the player. By removing impossible moves, like a person crossing a wall, we can keep the total uncertainty within an acceptable level in regions with physical obstacles. Additionally, it might be useful to introduce support for TCP communication to accommodate other use-cases that need to assure reliable message delivery.

As an alternative, the developer could eventually adopt a "seamful" design approach by adapting the game mechanics and bringing these limitations into the game itself. In some cases, informing the player about the existence of problems and exploiting them as game features, can constitute a valid alternative [10.

The present method can be used as a stand-alone solution, or as an auxiliary component of another location technology, which makes it also useful for outdoor-only games that require higher precision than a GPS-only solution can provide. Nevertheless, other location-aware products and services can also gain from the use of these techniques. A migration to a smartphone platform and a more intensive end-user experience would definitely foster further refinements and help consolidating the solution.

Acknowledgments. This work is partially supported by the Portuguese government, through the National Foundation for Science and Technology - FCT (Fundação para a Ciência e a Tecnologia) and the European Union (COMPETE, QREN and FEDER) through the project PTDC/EIA-EIA/114868/2009 entitled "Eras - Expeditious Reconstruction of Virtual Cultural Heritage Sites".

\section{References}

1. Greenfield, A.: Everyware: the dawning age of ubiquitous computing. New Riders (2006)

2. Levi, R., Judd, T.: Dead reckoning navigational system using accelerometer to measure foot impacts. U.S. Patent Number 5, 583, 776 (1996) 
3. Syrjärinne, J., Käppi, J., Saarinen, J.: MEMS-IMU based pedestrian navigator for handheld devices. Nokia Research Center (2001)

4. Liu, H., Darabi, H., Banerjee, P., Liu, J.: Survey of Wireless Indoor Positioning Techniques and Systems. IEEE Transactions on Systems, Man, and Cybernetics, Part C: Applications and Reviews (2007)

5. Koyuncu, H., Yang, S.: A Survey of Indoor Positioning and Object Locating Systems. IJCSNS International Journal of Computer Science and Network Security 10(5) (2010)

6. Wang, A., Jarrett, M., Sorteberg, E.: Experiences from implementing a mobile multiplayer real-time game for wireless networks with high latency. Hindawi Publishing Corp. (2009)

7. Premerlani, W., Bizard, P.: Direction Cosine Matrix IMU: Theory (2009), gentlenav.googlecode.com/files/DCMDraft2.pdf

8. Ladetto, Q., Merminod, B.: Digital Magnetic Compass and Gyroscope Integration for Pedestrian Navigation. In: 9th Saint Petersburg International Conference on Integrated Navigation Systems (2002)

9. Chen, K.-T., Huang, C.-Y., Huang, P., Lei, C.-L.: An empirical evaluation of TCP performance in online games. In: Proceedings of the 2006 ACM SIGCHI International Conference on Advances in Computer Entertainment Technology. ACM (2006)

10. Broll, G., Benford, S.: Seamful Design for Location-Based Mobile Games. In: Kishino, F., Kitamura, Y., Kato, H., Nagata, N. (eds.) ICEC 2005. LNCS, vol. 3711, pp. 155-166. Springer, Heidelberg (2005) 